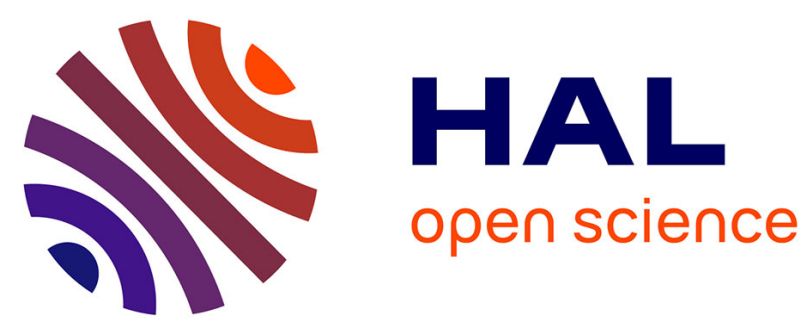

\title{
NON LOCAL PINNING AND INVERSION TEMPERATURE IN INHOMOGENEOUS SUPERCONDUCTORS
}

L. Dobrosavljevic, Z. Radovic, M. Kulic

\section{- To cite this version:}

L. Dobrosavljevic, Z. Radovic, M. Kulic. NON LOCAL PINNING AND INVERSION TEMPERATURE IN INHOMOGENEOUS SUPERCONDUCTORS. Journal de Physique Colloques, 1978, 39 (C6), pp.C6-646-C6-647. 10.1051/jphyscol:19786290 . jpa-00217732

HAL Id: jpa-00217732

https://hal.science/jpa-00217732

Submitted on 1 Jan 1978

HAL is a multi-disciplinary open access archive for the deposit and dissemination of scientific research documents, whether they are published or not. The documents may come from teaching and research institutions in France or abroad, or from public or private research centers.
L'archive ouverte pluridisciplinaire HAL, est destinée au dépôt et à la diffusion de documents scientifiques de niveau recherche, publiés ou non, émanant des établissements d'enseignement et de recherche français ou étrangers, des laboratoires publics ou privés. 


\section{NON LOCAL PINNING AND INVERSION TEMPERATURE IN INHOMOGENEOUS SUPERCONDUCTORS}

L. Dobrosavljevic, Z. Radovic and M. Kulic

Institute of Physics, P.0. Box 57, IZool Beograd, Yugoszavia

Résumé.- Le piégeage des vortex dans un superconducteur inhomogène à structure périodique n'est "local" qu'au dessous de 1 a "température d'inversion" $T^{\text {*h }}$. Pour $T>T$, la variation du courant critique en fonction de la température n'est plus monotone, grâce aux effets non-locaux.

Abstract.- Pinning in a type II superconductor with periodically distributed inhomogeneities is "local" only below the "inversion temperature" $T^{*}$. For $T>T^{*}$, due to nonlacal pinning of vortices, the critical current nonmonotonically varies with the temperature change.

The pinning of vortices in "layered" superconductors with regular distribution of inhomogeneities is known to give rise to a nonmonotonic variation of critical current density $j_{c}$ in function of the external field/1,2/. If one considers a "layered" type of inhomogeneity, with homogeneous subdomains repeated periodically, the pinning is governed by "local" properties of these subdomains only if their dimensions are large compared to the size of a vortex. The effect of nonlocality leads to a nonmonotonic variation of $j_{c}$ with the temperature change, as shown in figure 1.

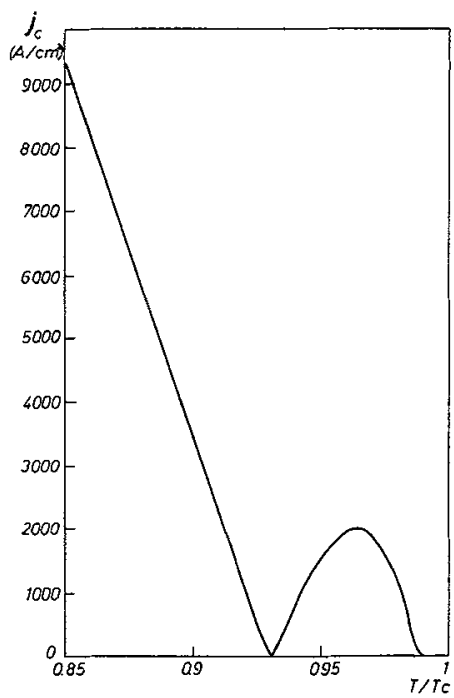

Fig. 1.: Temperature dependence of the critical current density $j_{c_{0}}$ presented for $\Delta d / d=0,1$; $\overline{\mathrm{d}}=10^{3} \AA ; \mathrm{L}_{\mathrm{o}}=10^{4} \AA ; \bar{l}=50 \AA ; \mathrm{K}=5 ; \bar{\xi}=10^{3}$ $\left(1-T / T_{C}\right)^{-i / 2} \AA$

In periodic structure such as :

a) Thin films with periodically modulated thickness in a perpendicular magnetic field/2/;

b) Thick alloy films with periodically modulated concentration in a field parallel both to the film and to the equiconcentration planes/1/; the effect of spatial variations of the electronic mean free path $\&$ on pinning is considered.

In the low field domain, $\mathrm{H} \gtrsim_{\sim} \mathrm{H}_{\mathrm{cl}}$, the pinning is governed by the variation $\Delta \varepsilon$ of the vortex self-energy $\varepsilon$ calculated using the Ginzburg-Landau (GL) theory extended to include the inhomogeneous case $/ 3 /$.

Let us firstly consider a thin film of thick ness modulated in a given direction, $d(x)=\bar{d}+$ $\Delta d \cos \frac{2 \pi}{L}\left(x-x_{0}\right)$ in a perpendicular field. Assuming that $\bar{l}<<\overline{\mathrm{d}}<<\bar{\xi}_{0}$, where $\bar{l}$ is the mean free path for the bulk scattering, we find a Pearl's-like solution of the GL equations valid for $\mathrm{K} \gg>1, \lambda_{\text {eff }}=$ $\bar{\lambda}^{2} / \bar{d} \gg \bar{\xi}$ and $\bar{d} / 4 \lambda_{\text {eff }}<<1$. The resulting electromagnetic contribution to $\Delta \varepsilon$, calculated in the $1^{\text {st }}$ order in $\Delta d / \bar{d}$, is given by

$\Delta \varepsilon_{\mathrm{M}} \cong \frac{\Delta \mathrm{d}}{\overline{\mathrm{d}}}\left(\frac{\phi_{0}}{4 \pi}\right)^{2} \frac{1}{\lambda_{\text {eff }}}\left[\ln \frac{4 \lambda_{\text {eff }}}{\gamma \bar{\xi}}-0.5\right] \mathrm{J}_{\mathrm{o}}\left(\frac{2 \pi \bar{\xi}}{\mathrm{L}}\right) \cos \frac{2 \pi \mathrm{x}_{\mathrm{o}}}{\mathrm{L}}$

where $J_{0}$ is the Bessel function and $\gamma=1.781$ is the Euler constant. The core contribution $\Delta \varepsilon_{c}$ varies with temperature in a similar way/3/. Furthermore, the long-range electromagnetic interaction, that is characteristic for Pearl's vortices, allows us to adopt that $\Delta \varepsilon \approx \Delta \varepsilon_{M}$. At low $T$, where $\bar{\xi}(\mathrm{T})<<\mathrm{L}$, $\mathrm{J}_{0}(2 \pi \bar{\xi} / \mathrm{L})$ is positive and the energy minimum corresponds to the pinning of vortices by the thin regions $\left(\cos \frac{2 \pi x_{0}}{\mathrm{~L}}=-1\right)$. The pinning is no more "1ocal" for temperatures higher than the "inversion" temperature $\mathrm{T}_{1}^{\mathbf{K}}$, at which $\Delta \varepsilon=0$ for the first time. Consequently, $\bar{\xi}\left(\mathrm{T}_{1}^{*}\right) \approx 0.4 \mathrm{~L}$. For $\mathrm{T}_{2}^{\mathrm{F}}>\mathrm{T}>\mathrm{T}_{1}^{\mathrm{F}}$, Jo is negative, and the centers of vortices are pinned by 
the thickness maxima $\left(\cos \frac{2 \pi x_{0}}{L}=1\right)$. Such an anusual temperature dependence of $\Delta \varepsilon$ is experimentally evidenced in a nonmonotonic variation of the critical current density in a "matching" configuration $\mathrm{j}_{\mathrm{cM}}=\frac{c}{\phi_{0}} \frac{2 \pi}{\mathrm{L}}\left|\frac{\Delta \varepsilon}{\mathrm{d}}\right|$ (figure 1). A very good agreement

is obtained between the theoretical and experimental results $/ 4 /$.

Similar results are obtained for an alloy with a modulated concentration $n(x)=\bar{n}+\Delta n \cos \frac{2 \pi}{L}$ $\left(x-x_{0}\right)$, in a parallel field. When both core and electromagnetic contributions are considered, we get in the $1^{\text {st }}$ order in $\Delta \mathrm{n} / \overline{\mathrm{n}}$

$$
\begin{aligned}
\Delta \varepsilon \cong- & \frac{\Delta \mathrm{n}}{\overline{\mathrm{n}}}\left(\frac{\phi_{0}}{4 \pi}\right)^{2} \frac{1}{\bar{\lambda}^{2}}\left\langle\mathrm{~J}_{\mathrm{O}}\left(\frac{2 \pi \bar{\xi}}{\mathrm{L}}\right)\left[\ln \frac{2 \bar{\lambda}}{\gamma \bar{\xi}}-0.5\right]+0.25\right\} \\
& \cos \frac{2 \pi \mathrm{x}_{\mathrm{o}}}{\mathrm{L}}
\end{aligned}
$$

At low $T$, the expression in parentheses is positive, and the vortices are pinned "locally" by the high concentration regions. The "inversion" temperature $\mathbf{T}_{j}^{\mathbf{X}}$, defined by the first zero of $\Delta \varepsilon$, is somewhat higher than in the previous case. For $\mathrm{T}_{2}^{*}>\mathrm{T}>\mathrm{T}_{1}^{*}$, the concentration minima act as pinning centers. More precise numerical solution of the GL equations provide the variation of the vortex selfenergy with the vortex position and temperature, as per figure 2 . In the present case $\bar{\xi}\left(T_{1}^{*}\right) \approx 0.5 \mathrm{~L}$.

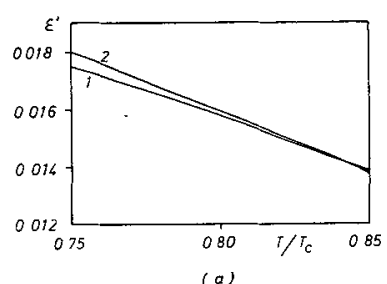

(a)

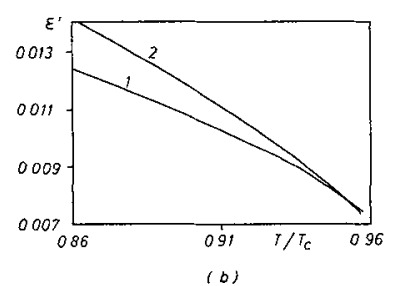

(b)
Fig. 2 : Temperature dependence of reduced free energy per unit length $\varepsilon^{\prime}\left(\mathrm{T} / \mathrm{T}_{\mathrm{C}}\right)=\varepsilon\left(\mathrm{T} / \mathrm{T}_{\mathrm{C}}\right) / \mathrm{H}_{\mathrm{C}}^{2}(0) \lambda^{2}(0)$ for a single vortex with $K=5$ and (a) $L / \lambda(0)=1$, (b) $\mathrm{L} / \lambda(\mathrm{o})=2$. The curves (1) calculated for the vortex center positioned in the region of the maximum concentration of impurities. The curves (2) for the vortex center positioned in the region of the minimum concentration of impurities.

In the vicinity of $\mathrm{H}_{\mathrm{C} 2}$, the effect of nonlocality is manifested through both the critical current and the critical field dependence on temperature. The transition curves $\mathrm{H}_{\mathrm{c} 2}^{\prime \prime}(\mathrm{T})$ for a modulated alloy show/5/ two characteristic temperatures $\mathrm{T}_{1}^{\mathrm{*}}$ and $\mathrm{T}_{2}^{*}$. The "inversion" temperature $\mathrm{T}_{1}^{* \mathrm{~K}}$ is the distinguishing point between the temperature range where the vortices nucleate in the concentration maxima $\left(\mathrm{T}<\mathrm{T}_{1}^{*}\right)$ and that where the nucleation occurs in the concentration minima $\left(\mathrm{T}>\mathrm{T}_{1}^{*}\right)$. Ami and Maki/6/ performed a calculation of $j_{c}$ for the same system, and found that $j_{c}=0$ at $T=T_{1}^{*} \cdot T_{1}^{*}$ is the temperature at which the core of a vortex covers only half a period of modulation, i.e. $\bar{\xi}\left(\mathrm{T}_{\mathrm{I}}^{*}\right) \approx 0.25 \mathrm{~L}$, while at $\mathrm{T}_{2}^{* \mathrm{~K}}\left(\bar{\xi}\left(\mathrm{T}_{2}^{* *}\right) \approx 0.5 \mathrm{~L}\right)$, the vortex core overlaps the whole period. For $T>T_{2}^{*}$, the transition curve $\mathrm{H}_{\mathrm{c} 2}(\mathrm{~T})$ for the modulated alloy has a positive curvature, which reflects a change from a $2 d-1$ ike to a $3 d-1 i k e$ behaviour of a layered-type system/5,7/.

Finally, one should notice that the observed effect may lead to the production of samples that are much harder superconductors in the vicinity of the transition temperature.

\section{References}

/1/ Raffy,H., Guyon,E. and Renard,J.C., Solid State Commun. 14 (1974) 872

/2/ Martinoli,P., Daldini,0., Leemann,C. and Stockes,E., Solid State Commun. 17 (1975) 205 Martinoli,P., Daldini,D., Leemann,C. and Van der Brandt,B., Phys. Rev. Lett. 36 (1976) 382

13/ Radovic,Z., Dobrosavljevic,L. and Kulic,M., to be published in Solid State Commun.

14/ Daldini,0., private communication

15/ Dobrosavljevic,L, and Kulic,M., to be published in Journal of Low Temperature Physics 32 (1978) $n^{\circ} 3 / 4$

/6/ Ami,S. and Maki,K., Progr. Theor. Phys. 53 (1975) 1

/7/ Klemm,R.H. and Luther,A., Phys. Rev. B12(1975) 